Compte rendu de Engaging with Strangers: Love and Violence in the Rural Solomon Islands, de Debra McDougall

Jean-Louis Rallu

\title{
CpenEdition
}

Journals

Édition électronique

URL : http://journals.openedition.org/jso/7521

DOI : $10.4000 /$ jso.7521

ISSN : $1760-7256$

Éditeur

Société des océanistes

Édition imprimée

Date de publication : 31 décembre 2016

Pagination : $307-308$

ISSN : 0300-953x

Référence électronique

Jean-Louis Rallu, "Compte rendu de Engaging with Strangers: Love and Violence in the Rural Solomon Islands, de Debra McDougall », Journal de la Société des Océanistes [En ligne], 142-143 | 2016, mis en ligne le 31 décembre 2016, consulté le 24 septembre 2020. URL : http://journals.openedition.org/jso/ 7521 ; DOI : https://doi.org/10.4000/jso.7521

Ce document a été généré automatiquement le 24 septembre 2020.

(c) Tous droits réservés 


\section{Compte rendu de Engaging with Strangers: Love and Violence in the Rural Solomon Islands, de Debra McDougall}

Jean-Louis Rallu

\section{RÉFÉRENCE}

MCDOUGALL Debra, 2016. Engaging with Strangers: Love and Violence in the Rural Solomon Islands, New York, Oxford, Berghahn, 308 p., 24 illus., bibliogr., index.

1 L'auteure commençait tout juste son "terrain" à Ranongga quand éclata le conflit qui fut appelé "Ethnic Tension » en raison d'une mécompréhension de ses causes profondes. Dans les premiers chapitres, l'ouvrage présente une étude ethnographique «classique» puis montre les pratiques traditionnelles évoluant, altérées par leur confrontation avec les changements apportés par la colonisation, la christianisation, l'indépendance et le commerce international : mines et principalement logging, où se trouvent les principales causes du conflit qui agita les îles Salomon de 1998 à 2003.

2 L'est des îles Salomon est remarquable en raison de migrations traditionnelles très fréquentes. Le rôle culturel des migrants est une donnée fondamentale de la vie en société. Les mythes d'origine et les mythes fondateurs des clans montrent que les «humains " étaient des ogres ou des êtres absurdes avant de " commercer» (engage) avec des personnes venues d'au-delà de leurs côtes - à l'époque, principalement de la région insulaire centrée autour de la Nouvelle-Géorgie. La mort d'un chef s'accompagnait de sacrifices de victimes humaines capturées dans d'autres îles, mais aussi de la prise de captifs(ves) qui pouvaient être intégré(e)s dans le clan, entre autres, en vue d'éviter une éventuelle extinction de celui-ci. 
3 Si la terre est la propriété inaliénable des clans matrilinéaires (butubutu), l'accès à la propriété par des non-originaires était possible par des dons de bakia (larges et lourds cercles taillés et polis dans des bénitiers fossilisés). On trouve des bakia sur les sanctuaires ancestraux (tabuna) des clans autochtones, mais aussi sur ceux des clans immigrés. Ils symbolisent l'ancrage du groupe à la terre.

4 Il existait aussi, comme ailleurs dans le Pacifique, un droit à l'usufruit (secondary right) pour ceux qui avaient été autorisés à débrousser une parcelle, y planter des arbres ou faire des jardins ou des plantations, mais ce droit était pratiquement permanent, sauf en cas de conduite associable rompant avec les règles de l'hospitalité. Dans ces «transactions» anciennes, les propriétaires ne considéraient pas vendre un morceau de terre, mais «ancrer » sur leurs propres terres les «visiteurs» qui devenaient des invités et en quelque sorte faisaient partie de la communauté, voire de la «famille étendue » suite aux intermariages sur plusieurs générations.

$5 \mathrm{Vu}$ la facilité des migrations, une famille nucléaire pouvait s'établir, le plus souvent temporairement, sur les terres du mari, mais ses enfants ne pouvaient pas hériter de la propriété du sol qui passe par les femmes. Cependant, lorsque l'homme avait débroussé un espace, celui-ci passait à ses enfants, au détriment des lignées maternelles. Ceci fut encore facilité par l'orientation patriarcale associée à la Christianisation. En réaction, certains clans voulaient exiger une résidence matrilocale et uxorilocale.

6 Les achats de terres (pajuku) principalement en argent, mais incluant aussi parfois un ou plusieurs bakia, qui se sont développés récemment sont considérés comme non traditionnels. Ils ne peuvent que concerner l'usufruit, mais les acheteurs revendiquent aussi le droit à la terre. La pression démographique et la croissance rapide de la valeur économique des terrains ont conduit à ce que des "transactions" anciennes, essentiellement en bakia, se trouvent assimilées aux pajuku.

7 La colonisation - pour aliéner les terres -, puis les multinationales minières et du bois, et aussi les organisations internationales pour le développement se sont basées sur des concepts élémentaires et tronqués du droit du sol dans les sociétés traditionnelles ("pré-modernes »), et ont recherché les ayant droits " tribaux ». Ils ont ainsi exclu des populations qui s'étaient établies au fil des temps, il y a parfois plusieurs dizaines de générations, et multiplié les conflits fonciers, faits quasi inconnus auparavant. Les missions ont ajouté au bouleversement de la société traditionnelle en exacerbant ses tendances patriarcales: attaquant les domaines du ressort des matrilignages et réduisant le rôle "politique » des femmes qui pouvaient traditionnellement être chef de clan, en cas d'absence d'héritier mâle. -En une sorte de réponse, Joyce Dunaketo Panakera fonda la United Church Women's Fellowship en 1962. Des gouvernements post-coloniaux constitués uniquement d'hommes, corrompus et peu soucieux du développement socio-économique de leur population mirent la touche finale aboutissant à ce que la situation dégénère.

8 L'auteure présente une étude précise des droits sur le sol en relation au mythe et au peuplement de Ranongga qui apporte des informations nouvelles sur cet aspect essentiel des cultures du Pacifique, puis des méfaits de leur mécompréhension par les colonisateurs et ensuite les multinationales - plus récemment, aussi par les organisations internationales -. Elle montre une grande habilité à démêler les divers éléments qui aboutirent à l'état de fait qui régnait dans les années 1980 et 1990 et aux événements malheureux qui s'ensuivirent. Elle termine sur une réflexion sur le nouveau cosmopolitanisme à partir des traditions locales qu'elle a observées et met en 
face des concepts philosophiques européens centrés, depuis Kant, sur la notion d'état souverain. Si les îles Salomon pouvaient être qualifiées de "failed state», ce n'est pas en raison d'une incapacité à forger une unité à partir de la mosaïque ethnoculturelle traditionnelle - ces modes de peuplement et de gestion des terres se rencontrent également, à divers degrés, dans l'Ouest des îles Salomon et sont au contraire la raison pour laquelle le conflit ne fut pas plus meurtrier - mais en raison de l'incurie des gouvernements coloniaux et ensuite nationaux pour assurer un niveau de vie acceptable à l'ensemble de la population et gérer les migrations internes en relation au droit du sol. Quelques-uns s'enrichirent rapidement avec les (maigres) royalties du logging qu'on voulut bien leur donner, d'autres ne reçurent pratiquement rien et d'autres enfin n'avaient à vendre que leur force de travail. C'est un exemple venant à point, montrant que l'arrivée d'étrangers peut transformer la société " pour son bien » dans un contexte où ceux-ci sont considérés comme des égaux - voire des parents dans un cadre d'exogamie traditionnelle. 